\title{
Corneal epithelial thickness profile in dry-eye disease
}

\author{
Mohamed Abou Shousha ${ }^{1} \cdot$ Jianhua Wang ${ }^{1} \cdot$ George Kontadakis $\mathbb{D}^{1} \cdot$ William Feuer $^{1} \cdot$ Ana P. Canto ${ }^{1}$ \\ Rodrigo Hoffmann ${ }^{1} \cdot$ Victor L. Perez ${ }^{1,2}$
}

Received: 22 August 2018 / Revised: 13 March 2019 / Accepted: 4 July 2019 / Published online: 1 October 2019

(c) The Author(s), under exclusive licence to The Royal College of Ophthalmologists 2019

\begin{abstract}
Background/objectives To characterize and evaluate the use of corneal epithelial profile maps generated by an ultrahighresolution optical coherence tomography (UHR-OCT) in the diagnosis and management of dry-eye disease (DED).

Subjects/methods This prospective, interventional case-control study included 115 eyes of 71 subjects (52 DED and 19 controls) imaged using an UHR-OCT. Average, maximum, and minimum, range of corneal epithelial thicknesses were extracted from epithelial profile maps. Surface regularity was quantified using the range and variance of the epithelial thickness measured along a horizontal UHR-OCT scan. The variance of thickness measurements along a scan was named epithelial irregularity factor (EIF). Symptoms of 31 DED patients (55 eyes) were quantified by questionnaire and correlated to epithelial profile findings, fluorescein staining, tear breakup time, and Schirmer's test. Twenty-one DED eyes were administered autologous serum drops and follow-up UHR-OCT images were captured.

Results DED patients had a highly irregular corneal epithelial surface compared with controls. Epithelial thickness profile variance (EIF) and range were significantly higher in DED as compared with controls (5.79 vs. $0.77, p<0.001$ and 7.6 vs. $4.6 \mu \mathrm{m}, p<0.001$ ). Both parameters were highly significantly correlated with questionnaire scores (EIF: $r=0.778 ; p<$ 0.001 , range: $r=0.737 ; p<0.001)$. Follow-up showed a statistically significant reduction in epithelial thickness profile variance and range of treated patients $(p<0.001)$.

Conclusions DED patients have irregular epithelial surface that can be quantified using UHR-OCT generated CEP maps. Epithelial thickness profile range and EIF correlate accurately with patients' symptoms and could be used to follow-up patients and response to treatment.
\end{abstract}

\section{Introduction}

Dry-eye disease (DED) has become a major area of research as ophthalmologists have realized its high prevalence and significant impact on patients' quality of life [1-7]. However, a major limitation in improving the standard of care for DED patients is the inability to objectively and quantitatively diagnose DED and monitor response to treatment due to the multifactorial aetiology of the disease. Those current diagnostic techniques used in everyday clinical practice include ocular surface staining, tear

Victor L. Perez

Victor.perez@duke.edu

1 Bascom Palmer Eye Institute, Department of Ophthalmology, University of Miami, Miller School of Medicine, Miami, FL, USA

2 Foster Center for Ocular Immunology at Duke Eye Center, DUMC 38022351 Erwin Rd, Durham, NC, USA breakup time (TBUT), Schirmer's test and symptoms questionnaires; additional techniques such as measurement of tear osmolarity, assessment of tear film biomarkers and tear film interferometry are gradually being incorporated in patients' care $[2,8]$

Standardization of those techniques is a challenge. That makes it difficult to compare studies by different authors or even follow-up patients [3, 4, 6-8]. In addition to lacking well-defined cutoff values to standardize grading of patients' severity, most of the tests are invasive to the extent that they can bias the obtained measurements by the mechanical, chemical, or other stimulations inherent to those techniques. Furthermore, most of these tests are poorly associated with patients' subjective symptoms [5-7]. It has become evident that development of new diagnostic techniques that identify new variables in the ocular surface that correlate with patients' symptoms is critical for the advancement in the treatment of DED patients.

Epithelial thickness is an anatomical parameter that has been studied recently in dry-eye patients with the use of 
epithelial maps created by means of optical coherence tomography. Two previous studies have opposing results regarding the effect of DED on epithelial thickness, describing either a thinning in superior cornea or a thickening in central cornea, but both agreeing that there is an increased variation of thickness throughout the cornea of DED patients in comparison with the cornea of controls $[9,10]$.

Our group has recently developed a prototype custombuilt ultrahigh-resolution optical coherence tomography (UHR-OCT) to study the ocular surface and corneal tissue layers in a detailed noninvasive fashion [11, 12]. Other groups have demonstrated the usefulness of OCT for epithelial thickness mapping in keratoconus patients [13] and even tear film meniscus thickness measurement in DED patients $[9,10]$. In the present study we demonstrate the use of UHR-OCT, for the creation of corneal epithelial profile maps to microscopically characterize the ocular surface and to identify irregularities in the corneal epithelium of DED patients. Moreover, we demonstrate that quantification of those irregularities captured on the CEP maps provide a noninvasive qualitative and quantitative measure to diagnose DED.

\section{Materials and methods}

\section{Study population}

We enrolled subjects from a continuous cohort of patients visiting the ocular surface clinic of the Bascom Palmer Eye Institute, Miami, FL either for follow-up of DED or for primary evaluation. Patients were enrolled in study group if diagnosed as DED patients on examination by a cornea specialist. Diagnosis was based on self-reporting of ocular discomfort and an abnormal result of any of the following diagnostic tests, which could be performed interchangeably depending on each patient's symptoms: corneal fluorescein staining scores, TBUT, Schirmer's test or The Shihpai dryeye symptom questionnaire scores. Abnormal corneal fluorescein staining was defined as measurements $>1$ [5], while abnormal TBUT and Schirmer's $I$ tests were defined as measurements $\leq 10 \mathrm{~s}$ and $\leq 5 \mathrm{~mm}$, respectively. Newly diagnosed patients underwent a complete workup with all clinical tests (will be referred to as "complete workup subgroup"), and old diagnosis of patients on follow-up was reconfirmed by repeating previous diagnostic testing. Normal controls were considered the subjects that were not identified as DED patients by any of the aforementioned tests. Patients on topical medications other than preservative-free artificial tears and contact lens wearers were excluded. Patients with current diagnosis or history of any other ocular surface or corneal disease such as burn, ulcer, ocular surface neoplasia, recurrent erosions, epithelial basement membrane dystrophy etc., and patients with history of refractive surgery were not included in the study.

Written informed consent, approved by University of Miami Institutional Review Board (IRB), was obtained from all patients.

\section{Dry-eye tests}

For symptoms quantification, The Shihpai dry-eye symptom questionnaire was used [6]. It consists of eight questions that were scored and patients were allowed to choose either "Never", "Rarely = 1", "Sometimes =2", "Often $=3$ ", or "All the time $=4$ " as an answer. A total score is calculated by adding all questions' score. To assess the status of the tear film stability, fluorescein TBUT was obtained from all the examined eyes [5]. The intensity of corneal and conjunctival fluorescein staining was recorded for each eye using the NEI staining grid in which a score of $0-3$ is assigned to each of five corneal and six conjunctival regions. A total corneal and conjunctival staining score was obtained providing a maximum score of 15 and 18 for corneal and conjunctival staining, respectively [14, 15]. Finally, Schirmer's $I$ test without anesthesia was performed $[5,14]$. Diagnosis of DED was based on abnormal results if corneal fluorescein staining was $>1$, TBUT $\leq 10 \mathrm{~s}$, and Schirmer's $I$ test $\leq 5 \mathrm{~mm}$ [15]. Patients reporting one or more symptoms as often or all the time in the questionnaire were diagnosed as DED patients $[6,16]$.

\section{Ultrahigh resolution-anterior segment OCT}

Analysis of the ocular surface was performed using novel custom-built spectral domain anterior segment OCT with an ultrahigh resolution of $\sim 3 \mu \mathrm{m}[9,10]$. Briefly, UHR-OCT uses a three-module superluminescent diode light source (Broadlighter, T870-HP, Superlum diodes Ltd, Moscow, Russia) with a center wavelength of $870 \mathrm{~nm}$ and a full width at half maximum bandwidth of $188 \mathrm{~nm}$. The A-line (depth scan) rate of the OCT system was set to $24 \mathrm{kHz}$. Sensitivity was measured to be about $95 \mathrm{~dB}$ and the resolution of the system was $\sim 3 \mu \mathrm{m}$ in water or tissue with a refractive index of $\sim 1.39[9,10,17]$.

\section{Corneal epithelial profile maps}

UHR-OCT imaging was performed before the clinical tests to avoid any alterations of the ocular surface. Patients fixated on a target and immediately after the blink, a 32 frames radial image of their cornea was captured in $2.7 \mathrm{~s}$. Custombuilt software was used to analyse the captured images and create $3 \mathrm{~mm}$ central corneal two-dimensional epithelial profile maps in the horizontal meridian. The software user 
inserts reference points on the anterior and posterior surfaces of the epithelium. The software automatically identifies the hyper-reflective points adjacent to those selected reference points in order to identify the surfaces to be delineated. The user reviews the delineated surface and is allowed to put more points as needed to ensure the accuracy of delineation. After delineation of the anterior and posterior surfaces of the epithelium, the software creates a thickness profile of the epithelium at $0.1 \mathrm{~mm}$ intervals centered on the corneal vertex and presents the data in a spreadsheet. Average, range, and variance of the obtained thickness profile of each eye is then calculated in the central $3 \mathrm{~mm}$ of the cornea along the horizontal frames.

\section{Epithelial irregularity factor (EIF)}

To numerically characterize each eye based on the severity of epithelium irregularity as recorded by the software, we used the variance of thickness measurements as an index which we named EIF. To validate the measurement of EIF, we tested its reproducibility and operator dependency. For reproducibility, eight eyes of four patients with test-retest measurements were collected $3 \mathrm{~h}$ apart. To determine operator dependency, five eyes were measured by two different operators.

\section{Modulation of EIF by DED therapy}

To directly test if elevated EIF can be modified by treatment of patients with DED and therefore, could be used as a tool to monitor therapy, 11 DED patients (21 eyes) were treated with autologous serum tears $20 \%$ four times a day. Baseline and follow-up EIF and dry-eye symptom questionnaire scores were obtained at pretreatment and follow-up visits. Last follow-up was used for statistical analysis.

\section{Statistical analysis}

SPSS software (SPSS, IL, USA) was used. Clinical tests and EIF measurements were made on both eyes of patients contributing two eyes to the study. However, since questionnaire scores are patient level and not eye specific assessments, the two eyes of patients who contributed both eyes were averaged. Similarly, for patient who underwent treatment and has contributed both eyes to the follow-up study, their two eyes measurements were averaged. Comparisons were made between parameters describing epithelium thickness profile between patients with DED and normal controls.

In the complete workup subgroup correlation of UHR-OCT parameters with severity of DED as evaluated clinically and by questionnaire was assessed by means of Pearson correlation. Linear stepwise regression analysis was used to identify significant predictors of questionnaire response among clinical parameters (Shirmer's test, Fluorescein staining, TBUT), age, and EIF in this subgroup of patients.

To detect if corneal epithelial maps of severe DED patients are different than those with milder diseases, we divided the subjects of the complete workup subgroup according to their questionnaire scores to two subgroups. The first subgroup included those with severe DED who had questionnaire scores more than or equal to 12 , while the second subgroup with mild DED included those with scores less than 12. Comparisons of EIF were made between two subgroups. Receiver operating characteristic (ROC) curves were constructed for the assessment of EIF as a parameter for the diagnosis of DED and severe DED.

Finally, comparison of EIF values prior to and after DED treatment was assessed. $P$-values less than 0.05 were considered statistically significant. Values are presented as mean \pm standard deviation.

\section{Results}

The study included 85 eyes of 52 DED patients and 30 eyes of 19 controls. Demographics are included in Table 1.

\section{Corneal epithelial profile maps-qualitative characterization}

Our semi-automated technique allowed for delineating of the ocular surface of all studied corneas. Our analysis of the corneal epithelial profile maps showed that DED patients had an irregular ocular surface that can be readily detected by the UHR-OCT (Fig. 1). In control subjects, the epithelium surface profile was different, with a "smooth" pattern (Fig. 2).

\section{Quantitative evaluation of corneal epithelial profile maps}

Average of epithelial thickness profile did not differ significantly between DED patients and controls. Mean value of the average of epithelial thickness profile in DED group was $53 \mu \mathrm{m}$ (standard deviation [SD] 22) and among controls it was $54 \mu \mathrm{m}(\mathrm{SD}=0.3 \mu \mathrm{m}, p=0.847$, independent samples $t$-test). Range of epithelial thickness profile in each eye had a significant difference between DED patients and controls. Mean value of range of epithelial thickness profile in DED patients was $7.6 \mu \mathrm{m}(\mathrm{SD}=4.6)$ and in control group it was 2.7 ( $\mathrm{SD}=0.6, p<0.0001)$. EIF was also significantly different between groups. Mean value of EIF in DED patients was $5.79(\mathrm{SD}=4.19)$ and in controls it was $0.77(\mathrm{SD}=0.28, p<0.0001)$ 
Table 1 Patient demographics and measured OCT parameters

\begin{tabular}{|c|c|c|c|c|c|c|}
\hline \multirow{4}{*}{$n$} & \multirow{4}{*}{$\begin{array}{l}\text { Males } \\
\text { Females }\end{array}$} & \multicolumn{2}{|c|}{ Dry-eye group } & \multicolumn{2}{|c|}{ Control group } & \multirow[b]{4}{*}{$P$ value } \\
\hline & & 52 & 11 & 19 & 8 & \\
\hline & & & 41 & & 11 & \\
\hline & & \multicolumn{4}{|c|}{ Mean (standard deviation) } & \\
\hline \multicolumn{2}{|c|}{ Age } & \multicolumn{2}{|c|}{$60(14.7)$} & \multicolumn{2}{|c|}{$33(10.9)$} & $<0.001$ \\
\hline \multicolumn{2}{|c|}{ Average of epithelial thickness profile $(\mu \mathrm{m})$} & \multicolumn{2}{|c|}{$53.1(21.8)$} & \multicolumn{2}{|c|}{$53.7(3.6)$} & 0.85 \\
\hline \multicolumn{2}{|c|}{ Range of epithelial thickness profile $(\mu \mathrm{m})$} & \multicolumn{2}{|c|}{ 7.6(4.6) } & \multicolumn{2}{|c|}{$2.7(0.6)$} & $<0.0001$ \\
\hline \multicolumn{2}{|c|}{ Minimum of epithelial thickness profile $(\mu \mathrm{m})$} & \multicolumn{2}{|c|}{ 49.1(0.019.4) } & \multicolumn{2}{|c|}{$52.1(3.5)$} & 0.28 \\
\hline \multicolumn{2}{|c|}{ Maximum of epithelial thickness profile $(\mu \mathrm{m})$} & \multicolumn{2}{|c|}{$56.7(22.9)$} & \multicolumn{2}{|c|}{$54.9(3.5)$} & 0.58 \\
\hline \multicolumn{2}{|c|}{ EIF } & \multicolumn{2}{|c|}{$5.796(4.194)$} & \multicolumn{2}{|c|}{$0.768(0.277)$} & $<0.0001$ \\
\hline
\end{tabular}

EIF epithelial irregularity factor
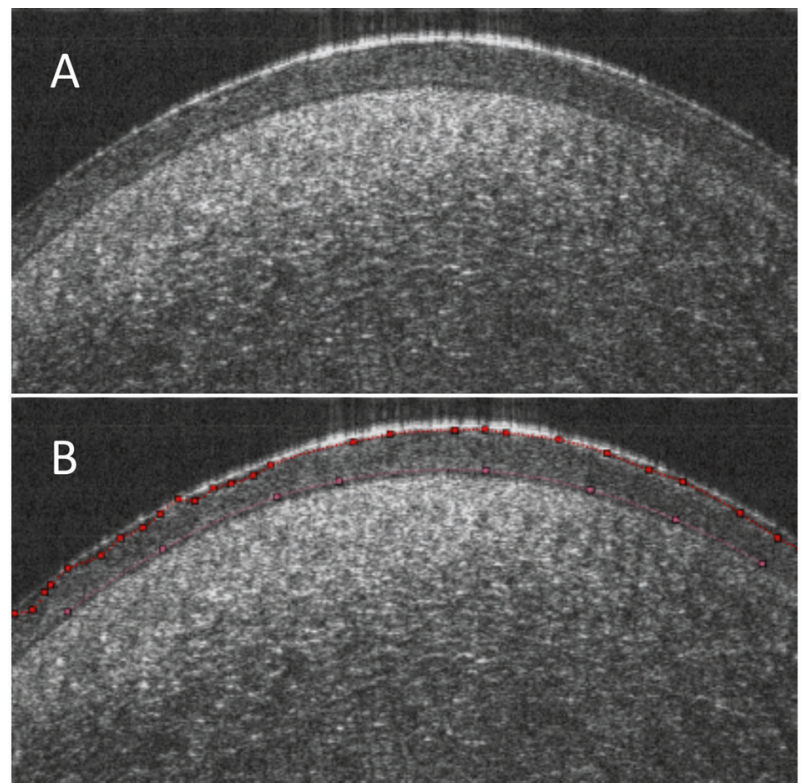

Fig. 1 Anterior corneal image of a patient with dry-eye disease obtained with the spectral domain anterior segment OCT with an ultrahigh resolution of $\sim 3 \mu \mathrm{m}$, $\mathbf{a}$ as acquired by the OCT, and $\mathbf{b}$ after inserting the reference points using the custom-built software in order to obtain the corneal epithelial profile map. The reference points are manually inserted by the user on the anterior (red dots) and posterior (purple dots) surface of the epithelium

\section{EIF validation}

Regarding reproducibility, we found an intraclass correlation coefficient of 0.94 (95\% confidence interval: 0.75-0.99), which is in the range usually considered good to excellent [16]. The coefficient of variation was also calculated for each pair of test-retest measurements and averaged was $12.5 \%$. Regarding operator dependency, the intraclass correlation coefficient between the two operators' measurements was 0.96 (95\% confidence interval: 0.91-1.00).

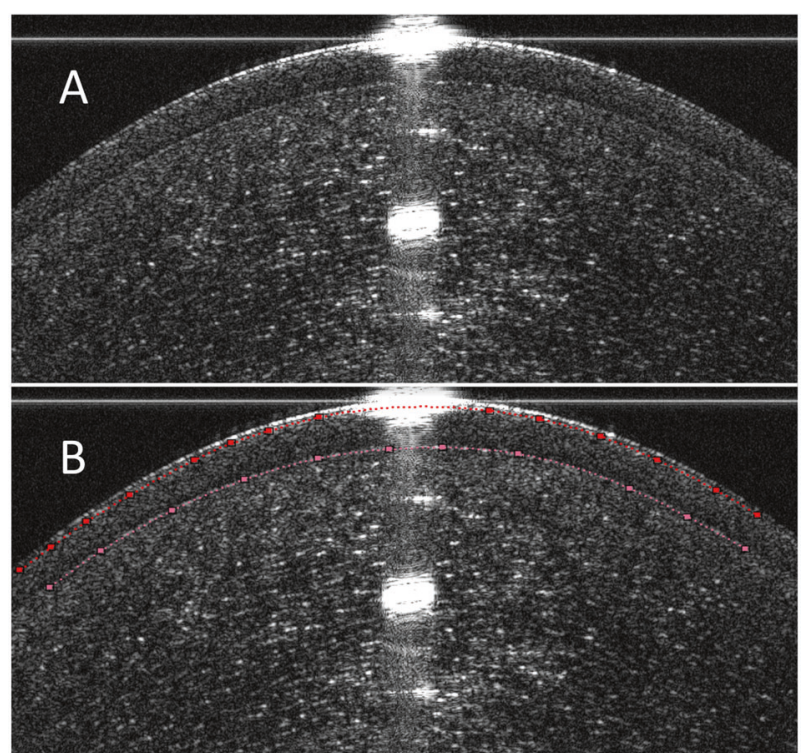

Fig. 2 Anterior corneal image of a control patient obtained with the spectral domain anterior segment OCT with an ultrahigh resolution of $\sim 3 \mu \mathrm{m}$, $\mathbf{a}$ as acquired by the OCT, and $\mathbf{b}$ after inserting the reference points using the custom-built software in order to obtain the corneal epithelial profile maps. The reference points are manually inserted by the user on the anterior (red dots) and posterior (purple dots) surfaces of the epithelium

\section{Correlation between EIF and DED Tests}

Fifty-five eyes of 31 newly diagnosed DED patients were included in the complete workup subgroup. Due to averaging of bilaterally measured patients, 31 sets of parameters were included in statistical analysis. EIF was significantly correlated with the questionnaire score, $(r=0.778, p<$ $0.0001)$ (Fig. 3) and with corneal Fluorescein staining $(r=$ $0.446, p=0.012$ ) (Fig. 4). A weaker but statistically significant correlation was found between EIF and Schirmer's test scores $(r=-0.375, p=0.038)$ (Fig. 4). Age was 
correlated with questionnaire score and EIF as well $(r=$ $-0.54, p=0.002, r=-0.446, p=0.012$, respectively). Partial correlation of EIF with the questionnaire scores was statistically significant when controlling for age $(r=0.713$, $p<0.001)$. Epithelial thickness profile range was also correlated with the score of the questionnaire $(r=0.737, p<$ $0.001)$ and weakly with the fluorescein staining of the cornea $(r=0.348, p=0.055)$. The questionnaire result, except of the EIF, was correlated only with the corneal fluorescein staining $(r=0.518, p=0.003)$. Correlations are included in Table 2. Multiple linear regression revealed as significant predictors of questionnaire score the EIF and TBUT $\left(r^{2}=0.688, p<0.001, p=0.011\right.$, respectively) (Table 3).

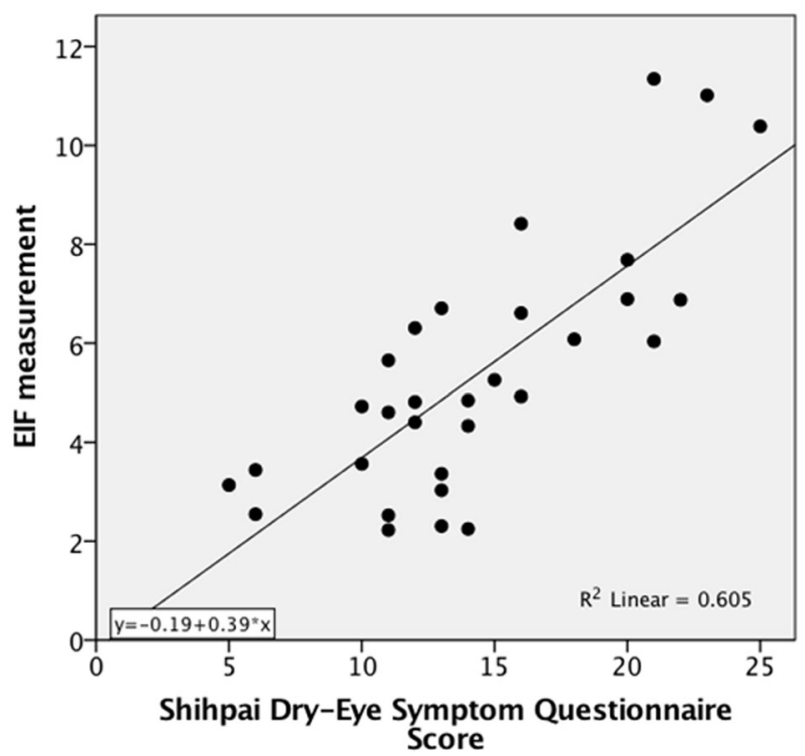

Fig. 3 Scatterplot of epithelial irregularity factor (EIF) measurement averaged between eyes versus the result of the Shihpai dry-eye symptom questionnaire score depicting the correlation in the group of patients with dry eye

\section{Mild versus severe DED}

EIF was significantly higher in severe DED patients as compared with patients with milder disease (6.08 vs. 3.60, $p=0.001)$. Other clinical parameters were also found to be more affected on average in the severe DED subgroup, but differences were not statistically significant (Schirmer's: $4.3 \mathrm{~mm}$ vs. $7.7 \mathrm{~mm}, p=0.4$, TBUT $4.4 \mathrm{sec}$ vs. $5.2 \mathrm{sec}$, $p=0.43$, corneal staining score 5.1 vs. $3.0, p=0.09$, conjunctival staining score 3.1 vs. $3.7, p=0.57$ ).

\section{EIF as a diagnostic tool}

In the complete group, the ROC area under the curve (AUC) for EIF was 1.00, $p<0.001$. A cutoff value of EIF larger than or equal to 1.345 had $100 \%$ sensitivity and $100 \%$ specificity in diagnosing DED in our group of patients. In diagnosis of severe DED in our subgroup of patients AUC of EIF was 0.813 ( $p=0.007)$. A cutoff value of EIF larger than or equal to 3.949 had $81.8 \%$ sensitivity and $77.7 \%$ specificity in diagnosing severe DED.

\section{Modulation of EIF by DED therapy}

Average follow-up of treated patients was 55 ( \pm 28$)$ days. There was a statistically significant reduction in thickness range from $8 \mu \mathrm{m}(\mathrm{SD}=2 \mu \mathrm{m})$ to $6 \mu \mathrm{m}(\mathrm{SD}=2 \mu \mathrm{m})(p<$ 0.001 , mixed model repeated measures analysis of variance) and in EIF from $7.75(\mathrm{SD}=4.94)$ to $3.73(\mathrm{SD}=2.86)$ in these eyes $(p<0.001$, paired samples $t$-test).

\section{Discussion}

In DED the protective function of tears is breached, and destruction of the ocular surface ensues. This results in the DED spectrum of signs and symptoms and can ultimately
Fig. 4 Scatterplots of epithelial irregularity factor (EIF) measurement versus a the result of Corneal Fluorescein Staining score and $\mathbf{b}$ the result of Schirmer's test depicting the correlation of EIF with both parameters
A

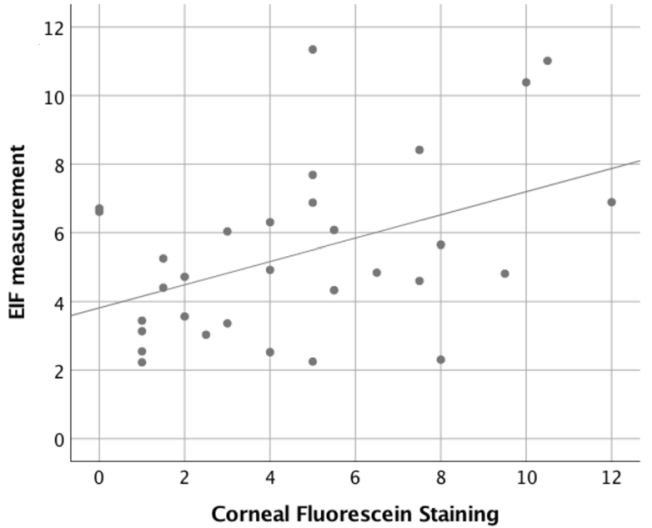

B

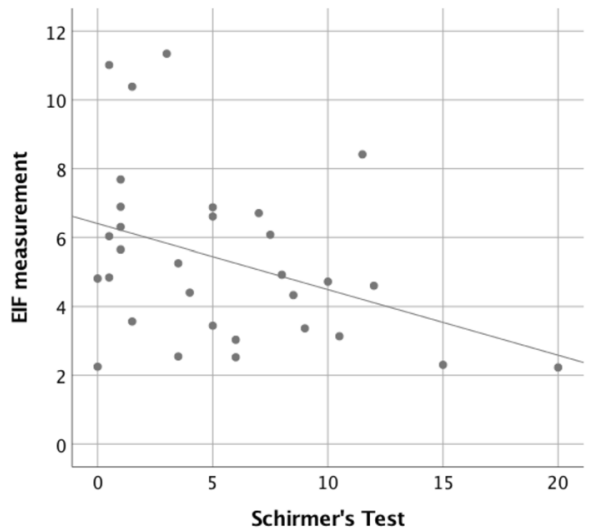


Table 2 Correlation between measured OCT parameters and clinical parameters in the complete workup subgroup of 31 patients

\begin{tabular}{|c|c|c|c|c|c|c|c|c|c|}
\hline & & EIF & $Q$ score & Schirmer's test & TBUT & $\begin{array}{l}\text { Corneal } \\
\text { Fluorescein } \\
\text { staining }\end{array}$ & $\begin{array}{l}\text { Conjunctival } \\
\text { Fluorescein } \\
\text { staining }\end{array}$ & $\mathrm{EpR} V+\mathrm{H}$ & Age \\
\hline \multirow[t]{2}{*}{ EIF } & Pearson's $r$ & 1 & $0.778 * *$ & $-0.375^{*}$ & 0.094 & $0.446^{*}$ & 0.176 & $0.904 * *$ & $-0.446^{*}$ \\
\hline & $P$ value & & 0.000 & 0.038 & 0.614 & 0.012 & 0.344 & 0.000 & 0.012 \\
\hline \multirow[t]{2}{*}{ Q score } & Pearson's $r$ & $0.778 * *$ & 1 & -0.334 & -0.215 & $0.518 * *$ & 0.091 & $0.737 * *$ & $-0.540 * *$ \\
\hline & $P$ value & 0.000 & & 0.066 & 0.246 & 0.003 & 0.625 & 0.000 & 0.002 \\
\hline \multirow{2}{*}{$\begin{array}{l}\text { Range of } \\
\text { epithelial } \\
\text { thickness } \\
\text { profile }\end{array}$} & Pearson's $r$ & $0.904 * *$ & $0.737 * *$ & -0.353 & 0.035 & 0.348 & 0.202 & 1 & $-0.470 * *$ \\
\hline & $P$ value & 0.000 & 0.000 & 0.051 & 0.853 & 0.055 & 0.277 & & 0.008 \\
\hline \multirow[t]{2}{*}{ Age } & Pearson's $r$ & $-0.446^{*}$ & $-0.540 * *$ & $0.439^{*}$ & $0.432 *$ & -0.304 & -0.225 & $-0.470 * *$ & 1 \\
\hline & $P$ value & 0.012 & 0.002 & 0.013 & 0.015 & 0.096 & 0.223 & 0.008 & \\
\hline
\end{tabular}

Q score: Shihpai dry-eye symptom questionnaire score

EIF epithelial irregularity factor, TBUT tear breakup time

**Correlation is significant at the 0.01 level (two-tailed).

*Correlation is significant at the 0.05 level (two-tailed).

Table 3 Multiple regression analysis for the predictors of Shihpai dryeye symptom questionnaire results

Dependent variable: Shihpai dry-eye symptom questionnaire results

\begin{tabular}{lccc}
\hline & \multicolumn{2}{l}{ Unstandardized coefficients } & $p$ value \\
\cline { 2 - 3 } & $B$ & Std. Error & \\
\hline (Constant) & 8.011 & 1.458 & 0.000 \\
EIF & 1.615 & 0.213 & 0.000 \\
TBUT & -0.500 & 0.182 & 0.011 \\
$R^{2}=0.688$ & & &
\end{tabular}

EIF epithelial irregularity factor, TBUT tear breakup time

lead to loss of the integrity of the eye [15]. DED diagnostic techniques could rely on detecting the abnormal tear film such as in Schirmer's test or TBUT. Studies have shown poor association between those tests and patients' symptoms [5, 7]. Many of those tests are poorly standardized since they are affected by factors that are difficult to control, such as variability in dye concentration and amount of illumination in surface-staining techniques, lack of standardization in grading etc. [3, 4]. Schirmer's test is invasive and unpleasant to the patient $[18,19]$. Other tests used are ocular surface staining, which is also invasive due to the instillation of a dye, and more recently, tear film osmolarity which has been established as a quantitative method to evaluate DED.

DED is a multifactorial disease that is affected by several parameters difficult to efficiently control, standardize, or quantify. A stepwise approach could include focusing on the effect and injury occurred could thus be a successful technique. New imaging modalities have been recently introduced such as confocal microscopy [20] and OCT [21].
Nevertheless, diagnosis of DED using confocal microscopy is a time-consuming procedure that can only capture images at a small area of the total cornea, and mostly requires contact with the ocular surface which makes it difficult to incorporate to everyday clinics. In vivo imaging of the ocular surface using OCT is a noninvasive and sensitive test. In the diagnosis of aqueous deficient DED, Shen et al. [21] has shown that by using OCT, the tear menisci can be measured and compared with normal. However, a limitation of this test was the ability to achieve standardization, as tear film is a dynamic element that is affected momentarily by several factors.

Using our novel UHR-OCT, we have examined the ocular surface down to a $3 \mu \mathrm{m}$ resolution which enabled us to disclose the injurious effect of DED and to quantify it. We have found that patients with DED have irregular ocular surface while normal subjects have a smoother surface. Even though we cannot directly test what EIF represents, we believe that an irregularity is most probably a manifestation of the injurious effect of dryness on the ocular surface which is translated by rich corneal sensory nerves into pain and discomfort. We have formulated a factor to describe the observed ocular surface irregularities and named it EIF. Our results have disclosed a highly statistically significant difference between EIF of normal subjects and DED patients. This is evidence that EIF is a novel qualitative criterion to diagnose DED.

Several of the currently available techniques, such as TBUT and fluorescein staining scores, have shown poor reproducibility and a significant operator dependency $[3,5,7]$. Testing the reproducibility and operator dependency of EIF was thus very important to validate it and to explore if it will provide an advantage over those classical 
techniques. A previous study has already demonstrated that epithelial thickness profile mapping by UHR-OCT has a high repeatability [22]. In the current study, although a small number of subjects were included for the reproducibility testing, we found that EIF has a very good reproducibility and has also shown minimal operator dependency, two major powerful points that would allow EIF to stand among other DED diagnostic techniques. Other studies have shown also a good reproducibility of OCT epithelial thickness mapping in healthy and diseased corneas [23, 24].

The discrepancy between DED symptoms and signs is a major limitation of current diagnostic techniques [7, 25]. The novel parameter that we present describes microscopic irregularities of the ocular surface and has demonstrated high correlation to DED patients' symptoms better than all other tested measurements. In the mathematical model created here to detect which of the tested values would best explain the variance in DED patients' symptoms, only EIF and TBUT were involved and explained $68 \%$ of patients' symptoms. Corneal fluorescein staining, despite showing significant correlation to patients' symptoms by itself, has failed to enter the model. This means that EIF and corneal fluorescein staining scores are actually measuring the same parameter, which is the injurious effect of DED on the ocular surface, and that EIF is more descriptive of that parameter.

The diagnostic value of our parameter is very high as well. In our group, it demonstrated an excellent AUC result, and the cutoff value for diagnosing DED had 100\% sensitivity and specificity. For the diagnosis of severe versus mild DED, AUC was satisfactory, and our cutoff value had $81.8 \%$ sensitivity and $77.7 \%$ specificity. Thus, EIF can serve to the diagnosis of DED and to distinguish patients with severe disease as well. We decided to divide our patients in subgroups of severe and nonsevere DED based on questionnaire results and not clinical test results since previous studies have shown a good correlation of questionnaire results with disease severity, and poor repeatability of clinical tests [25, 26].

Other authors had studied epithelial thickness parameters and their correlation to DED. Kanellopoulos and Asimelis [10] demonstrated that epithelial thickness was thicker in DED patients than controls, and that there are differences in the other parameters as minimum thickness, maximum thickness, and thickness variability as measured by their OCT. A superior thinning of the epithelium was also found in a study by Cui et al. [9]. In our study we did not find any differences in epithelial thickness, but we found a difference in range and variance of thickness throughout an image between DED and control patients. We decided to use thickness variation as a measure of DED severity and test its value within this study, since it is less affected by outliers, unlike range, minimum and maximum thickness and it has shown an excellent correlation to our questionnaire.

The search for new DED treatment modalities is hindered by the unavailability of an objective and sensitive technique that can explain better patients' symptoms. Lack of standardization and objectivity, poor repeatability and discordance between signs and symptoms make it difficult to detect patients' response to treatment using the current techniques [3, 4]. Our group has explored if EIF could be used to objectively monitor patients' response to treatment. Follow-up of DED patients treated with serum tears eye drops which is a treatment usually reserved for severe cases, has revealed that EIF was altered and shifted towards the normal value. This is evidence that EIF can be used to monitor patients and detect their response to treatment. A larger comparative randomized cohort of DED patients utilizing also other treatment methods is needed to confirm this.

Our study is not without limitations. The semi-automated method to compute EIF from UHR-OCT images is a limitation that makes calculating EIF a time-consuming procedure. A fully automated method to compute EIF would be a perfect means to efficiently incorporate EIF to the standard of care of DED. Our blinded validation pilot studies have shown very good operator dependency and very good repeatability. Nevertheless, it is important to mention that the reader was only blinded in those validation studies and not to all other cases included in the study. Another limitation in our study was the fact that the operator was allowed to set objective reference points until he/she determines that the correct surface is accurately delineated. Until a full automated smart code is developed, the need for a trained operator will be a requisite. Future studies should explore the specificity of EIF for the diagnosis of DED by studying the ocular surface irregularities in different other corneal pathological condition.

In summary, EIF measurements obtained using UHROCT is representative of the structural abnormalities imposed by DED on the ocular surface and it could be an objective means to qualitatively and quantitatively diagnose and follow-up DED. EIF is an accurate indicator of the clinical symptoms of the DED patients. EIF was able to quantitatively monitor patients' response to treatment and that makes it a powerful tool to help advancement of the standard of care of DED patients.

\section{Summary}

\section{What was known before}

- Dry-eye disease can be diagnosed with several clinical tests, but correlation with patients symptoms is poor. 


\section{What this study adds}

- Imaging of epithelial profile with ultrahigh resolution OCT and extrapolation of epithelial thickness variance can offer a reliable index for diagnosis of dry-eye disease, evaluate severity, and follow-up treated patients.

Funding This study was supported by NEI K23 award K23EY026118 (MAS), NEI core center grant to the University of Miami (P30 EY014801), and NEI R01EY018624 (VLP) grant from the National Eye Institute, unrestricted grant from Research to Prevent Blindness, NY and a grant from Alcon Research Ltd. The funding organizations had no role in the design or conduct of this research.

\section{Compliance with ethical standards}

Conflict of interest VLP is a consultant of Alcon Ltd. MAS and VLP are equity holders and sit on the Board of Directors for Resolve Ophthalmics, LLC. They are co-inventor of intellectual property licensed to Resolve Ophthalmics. PCT/US2018/013409, and US Patent No 8992023. VLP, MAS, and JW are co-inventor of NonProvisional United States Patent Application No.: 61809518.

Publisher's note Springer Nature remains neutral with regard to jurisdictional claims in published maps and institutional affiliations.

\section{References}

1. O'Brien PD, Collum LM. Dry eye: diagnosis and current treatment strategies. Curr Allergy Asthma Rep. 2004;4:314-9.

2. Sullivan BD, Whitmer D, Nichols KK, Tomlinson A, Foulks GN, Geerling G, et al. An objective approach to dry eye disease severity. Investig Ophthalmol Vis Sci. 2010;51:6125-30.

3. Foulks GN. Challenges and pitfalls in clinical trials of treatments for dry eye. Ocul Surf. 2003;1:20-30.

4. Yokoi N, Komuro A. Non-invasive methods of assessing the tear film. Exp Eye Res. 2004;78:399-407.

5. Pflugfelder SC, Tseng SC, Sanabria O, Kell H, Garcia CG, Felix $\mathrm{C}$, et al. Evaluation of subjective assessments and objective diagnostic tests for diagnosing tear-film disorders known to cause ocular irritation. Cornea. 1998;17:38-56.

6. Lin PY, Tsai SY, Cheng CY, Liu JH, Chou P, Hsu WM. Prevalence of dry eye among an elderly Chinese population in Taiwan: the Shihpai Eye Study. Ophthalmology. 2003;110:1096-101.

7. Nichols KK, Nichols JJ, Mitchell GL. The lack of association between signs and symptoms in patients with dry eye disease. Cornea. 2004;23:762-70.

8. Zeev MS-B, Miller DD, Latkany R. Diagnosis of dry eye disease and emerging technologies. Clin Ophthalmol. 2014;8:581-90.

9. Cui X, Hong J, Wang F, Deng SX, Yang Y, Zhu X, et al. Assessment of corneal epithelial thickness in dry eye patients. Optom Vis Sci. 2014;91:1446-54.
10. Kanellopoulos AJ, Asimellis G. In vivo 3-dimensional corneal epithelial thickness mapping as an indicator of dry eye: preliminary clinical assessment. Am J Ophthalmol. 2014; 157:63-68.e2.

11. Shousha MA, Karp CL, Perez VL, Hoffman RH, Ventura R, Chang V, et al. Diagnosis and management of conjunctival and corneal intraepithelial neoplasia using ultra high resolution optical coherence tomography. Ophthalmology 2011;118:1531-7.

12. Shousha MA, Perez VL, Wang J, Ide T, Jiao S, Chen Q, et al. Use of ultra-high-resolution optical coherence tomography to detect in vivo characteristics of descemet's membrane in Fuchs' dystrophy. Ophthalmology. 2010;117:1220-7.

13. Temstet C, Sandali O, Bouheraoua N, Hamiche T, Galan A, El Sanharawi M, et al. Corneal epithelial thickness mapping using Fourier-domain optical coherence tomography for detection of form fruste keratoconus. J Cataract Refract Surg. 2015;41: 812-20.

14. The definition and classification of dry eye disease: report of the Definition and Classification Subcommittee of the International Dry Eye WorkShop (2007). Ocul Surf. 2007;5:75-92.

15. Lemp MA. Report of the National Eye Institute/Industry workshop on Clinical Trials in Dry Eyes. CLAO J. 1995;21:221-32.

16. Schein OD, Munoz B, Tielsch JM, Bandeen-Roche K, West S. Prevalence of dry eye among the elderly. Am J Ophthalmol. 1997; 124:723-8.

17. Lin RC, Shure MA, Rollins AM, Izatt JA, Huang D. Group index of the human cornea at 1.3-microm wavelength obtained in vitro by optical coherence domain reflectometry. Opt Lett. 2004; 29:83-85.

18. Khanal S, Tomlinson A, McFadyen A, Diaper C, Ramaesh K. Dry eye diagnosis. Investig Ophthalmol Vis Sci. 2008;49:1407-14.

19. Lucca JA, Nunez JN, Farris RL. A comparison of diagnostic tests for keratoconjunctivitis sicca: lactoplate, Schirmer, and tear osmolarity. CLAO J. 1990;16:109-12.

20. Zhang X, Chen Q, Chen W, Cui L, Ma H, Lu F. Tear dynamics and corneal confocal microscopy of subjects with mild selfreported office dry eye. Ophthalmology. 2011;118:902-7.

21. Shen M, Li J, Wang J, Ma H, Cai C, Tao A, et al. Upper and lower tear menisci in the diagnosis of dry eye. Investig Ophthalmol Vis Sci. 2009;50:2722-6.

22. Wu S, Tao A, Jiang $\mathrm{H}, \mathrm{Xu} \mathrm{Z}$, Perez V, Wang J. Vertical and horizontal corneal epithelial thickness profile using ultra-high resolution and long scan depth optical coherence tomography. PLoS ONE. 2014;20:e97962.

23. Ma JX, Wang L, Weikert MP, Montes de Oca I, Koch DD. Evaluation of the repeatability and reproducibility of corneal epithelial thickness mapping for a 9-mm zone using optical coherence tomography. Cornea 2019;38:67-73.

24. Sella R, Zangwill LM, Weinreb RN, Afshari NA. Repeatability and reproducibility of corneal epithelial thickness mapping with spectral-domain optical coherence tomography in normal and diseased cornea eyes. Am J Ophthalmol 2019;197:88-97.

25. Lin PY, Cheng CY, Hsu WM, Tsai SY, Lin MW, Liu JH, et al. Association between symptoms and signs of dry eye among an elderly Chinese population in Taiwan: the Shihpai Eye Study. Investig Ophthalmol Vis Sci 2005;46:1593-8.

26. Nichols KK, Mitchell GL, Zadnik K. The repeatability of clinical measurements of dry eye. Cornea 2004;23:272-85. 